\title{
REVISIONES
}

\section{Convergencia europea e internacionalización del Doctorado: panorámica sobre las tendencias de cambio en España}

\author{
European convergence and internationalization of Doctoral Studies: \\ An overview of trends towards change in Spain
}

\author{
Magdalena Jiménez Ramírez. \\ Facultad de Ciencias de la Educación, Universidad de Granada, España \\ Telf.: (34) 958249887. Correo electrónico: madji@ugr.es
}

\section{RESUMEN}

La adaptación de los estudios de doctorado al Espacio Europeo de Educación Superior ha supuesto todo un cambio normativo en España, no exento de dificultades, con la finalidad de armonizar esta etapa formativa con las directrices europeas. El Real Decreto 99/2011 establece una nueva estructura organizativa para el doctorado y detalla las directrices, condiciones y procedimientos que deben regular los nuevos estudios doctorales. En este trabajo describimos algunos de los cambios más significativos, entre otros, la novedad de la creación de las Escuelas de Doctorado, y presentamos una panorámica descriptiva de algunos datos derivados de la implementación de normativa sobre la configuración y el desarrollo de los nuevos estudios de doctorado. Como conclusión se establece que las transformaciones están siendo complejas a nivel de gestión, organización y funcionamiento, aunque han implicado un giro hacia una formación que cualifique en competencias investigadoras dentro de un entorno de internacionalización y movilidad europea.

Palabras clave: legislación, escuelas doctorales, programas de doctorado, competencias investigadoras, datos estadísticos oficiales.

\begin{abstract}
The adaptation of Doctoral studies to the European Higher Education Area has involved a complex regulatory change in Spain aiming at harmonizing this stage of training with European guidelines. Royal Decree 99/2011 established a new organizational structure for the Doctorate and sets out the guidelines, conditions and procedures that are to regulate the new Doctoral studies. In this paper we describe some of the most significant changes, including, among others, the novelty of the creation of Doctoral Schools, and, likewise, we present a succinct descriptive overview of some data resulting from the implementation of the regulations on the configuration and putting into practice of the new Doctoral studies. We conclude that these transformations are complex at the levels of management, organization and operation, although they have involved a move towards training that qualifies candidates in research competencies within a setting of internationalization and European mobility.
\end{abstract}

Key words: legislation, doctoral schools, doctoral programs, research competences, official statistical data. 


\section{INTRODUCCIÓN}

El año 2010 era la fecha estipulada para la adaptación de los estudios universitarios al Espacio Europeo de Educación Superior (EEES). Esta finalidad, auspiciada por la Declaración de Bolonia (1999) ${ }^{1}$, ha implicado una profunda reforma de los planes de estudios en las universidades en grado y en posgrado (Agencia Nacional de Evaluación de la Calidad y Acreditación [ANECA], 2008²; Vázquez, 2008), afectando a las estructuras de las titulaciones, a la organización de las materias, a las dinámicas metodológicas y docentes, a la construcción de un proceso de enseñanza-aprendizaje activo, y a la transformación de los procesos de evaluación (Huber, 2008), al intentar desarrollar una enseñanza superior competitiva y de calidad (Villa \& Ruiz, 2004).

Los estudios de doctorado, instituidos como el último ciclo de las enseñanzas oficiales universitarias (art. 37-38, LOU 6/2001, modificada por la LOU 4/2007), también se han transformado para adaptarse a los principios del espacio común europeo, con la finalidad de constituir una red de formación en investigación que permita el desarrollo de acciones comunes a nivel europeo (ANECA, 2009). Al ser el grado académico más alto, cumplen un papel fundamental, pues suponen un nexo esencial entre la docencia y la investigación, además de ser una herramienta básica de conexión entre la universidad y la sociedad (Castro et al., 2010). En consecuencia, este ciclo de formación universitario es muy relevante por la trascendencia que se le atribuye en la relación con la sociedad. De ahí la importancia de estudiar y analizar cuáles son los cambios que se han introducido en las políticas educativas nacionales (Haug, 2008), considerando las directrices del EEES y del Espacio Europeo de Investigación.

En España, la adaptación del doctorado a los planteamientos europeos ha implicado una alteración en la normativa básica de estos estudios. Este proceso ha sido burocrático y administrativamente complejo (Jiménez \& Sevilla, 2016), puesto que se han aprobado por el Gobierno hasta cuatro Reales Decretos (RD) distintos que han afectado al funcionamiento organizativo de los estudios doctorales ${ }^{3}$. Actualmente, el RD 99/2011 es el que regula los estudios universitarios de tercer ciclo, que son concretados por los estatutos de las universidades, y estipula las características básicas que deben regir el funcionamiento y desarrollo de la etapa doctoral.

Por ello, el objetivo de este artículo es analizar los cambios introducidos en los estudios de doctorado, que implican una reestructuración en su organización, así como en las directrices y en las finalidades de los estudios doctorales. La innovación principal es la configuración de las Escuelas de Doctorado desde las que se gestiona y coordina la actual

Puede consultarse en la siguiente dirección, http://eees.universia.es/documentos/reuniones-ministros-educacion-superioreuropea/

2 La ANECA, Agencia Nacional de Evaluación de la Calidad, es un organismo autónomo que tiene como objetivo contribuir a la mejora de la calidad del sistema de educación superior mediante la evaluación, certificación y acreditación de enseñanzas, profesorado e instituciones, con el fin de integrar el sistema universitario en el EEES. Está adscrita al Ministerio de Educación, Cultura y Deporte y ha sido creada por el artículo 8 de la Ley 15/2014, de 16 de septiembre, de racionalización del Sector Público y otras medidas de reforma administrativa, procedente de la Fundación Agencia Nacional de Evaluación de la Calidad y Acreditación en organismo público, que se configuró a partir de la Ley Orgánica 6/2001, de 21 de diciembre, de Universidades (LOU), en su artículo 31.

3 Se han ido sucediendo distintos Reales Decretos: RD 56/2005 (derogado); RD 1393/2007 (vigente, excepto el capítulo V., Enseñanzas de Doctorado, que está derogado); RD 99/2011 (vigente); RD 534/2013 (vigente), por el que se modifica RD 99/2011, RD 1393/2007 y RD 1892/2008. 
estructura organizativa de los programas de doctorado, que también se transforman para darles una orientación más centrada en la investigación y en la formación en competencias transferibles al ámbito social y empresarial. Además, se presenta un análisis detallado de algunos datos derivados de la implementación de la actual normativa doctoral y se concluye con algunos retos para avanzar en la consolidación de las directrices para los estudios de doctorado en las universidades españolas.

\section{REESTRUCTURACIÓN DEL DOCTORADO: MODIFICACIONES CLAVE EN CONVERGENCIA EUROPEA}

La LOU (4/2007) atribuye a los estudios de doctorado como finalidad "la especialización del estudiante en su formación investigadora dentro de un ámbito del conocimiento científico, técnico, humanístico o artístico" (art.38), incluyendo "la elaboración, presentación y aprobación de un trabajo original de investigación" (art.38), en un contexto en transformación donde se asignan a la universidad las funciones de la investigación y la transferencia de conocimientos (art.39).

Este precepto está desarrollado ampliamente en el RD 99/2011, atribuyendo al doctorado la premisa de que estos estudios conduzcan "a la adquisición de las competencias y habilidades relacionadas con la investigación científica de calidad" (art.2.1.). Para Benito, Gil y Romera (2014), los doctores altamente cualificados en investigación y producción científica y tecnológica representan un valor estratégico de primera magnitud para su inserción en el sector productivo. De ahí que la ordenación del doctorado está orientada a formar investigadores desde las universidades que generen capital formativo de alta cualificación que implique transferencia de conocimientos hacia la sociedad, todo ello con la finalidad de desarrollar los compromisos adquiridos por los ministros de educación de los estados miembros en las reuniones celebradas en diferentes ciudades europeas ${ }^{4}$.

Considerando esta finalidad atribuida al doctorado, se han acometido modificaciones relevantes en la regulación de la etapa, específicamente, la innovación en la creación de las Escuelas de Doctorado; la transformación de los programas de doctorado para especializar en la investigación; la necesidad de formar en competencias, sobre todo investigadoras y no solo docentes, que impliquen transferencia de conocimiento a un entorno socio-laboral; y la relevancia de la internacionalización en la formación investigadora. Seguidamente, se sintetizan los principales cambios.

\subsection{GESTIÓN ORGANIZATIVA A TRAVÉS DE LAS ESCUELAS DE DOCTORADO}

El cambio más relevante es la configuración de las Escuelas de Doctorado. Son entidades configuradas por una universidad o conjuntamente por varias, donde también se puede establecer colaboración con otras entidades, instituciones u organismos, tanto nacionales como internacionales, que desarrollan actividades de $\mathrm{I}+\mathrm{D}+\mathrm{i}$, cuya finalidad principal es la

\footnotetext{
Los ministros europeos de educación se han reunido en varias ocasiones para analizar el avance del EEES y los progresos conseguidos. De estos encuentros han surgido distintos comunicados (comunicado de Berlín, 2003; de Bergen, 2005; de Londres, 2007; de Lovaina, 2009; Budapest-Viena, 2010; Bucarest, 2012), donde se han determinado las condiciones básicas que debe presentar el doctorado en un entorno europeo de calidad en la investigación. Las declaraciones se pueden consultar en http://eees.universia.es/documentos/reuniones-ministros-educacion-superior-europea/
} 
gestión organizativa de un ámbito de conocimiento sobre los estudios de doctorado, en una o varias ramas de conocimiento, que pueden tener una perspectiva interdisciplinar (RD 99/2011, art.2.4.). Como estructura específica para el doctorado, se constituye como una unidad autónoma con competencia en investigación, con un liderazgo en su estructura para organizar y gestionar las actividades propias de la formación científico-investigadora en un ámbito de conocimiento (art.9). Junto a ello, otro objetivo es conformar lazos de colaboración con centros de investigación, desarrollo e innovación en el ámbito nacional pero, fundamentalmente, internacional.

Las Escuelas de Doctorado también intervienen en la gestión de la formación del doctorando, estableciendo los procedimientos y líneas investigativas para la elaboración de las tesis doctorales, donde la relevancia radica en la competencia investigadora de los doctores, que "han de jugar un papel esencial en todas las instituciones implicadas en la innovación y la investigación, de forma que lideren el trasvase desde el conocimiento hasta el bienestar de la sociedad" (art.9, p. 13.909).

Se instituye un Comité de Dirección, encargado de la organización y gestión de la Escuela de Doctorado, y una Comisión Académica, cuya función es programar los estudios doctorales atendiendo a la formación y a la investigación, en concreto, asignación de tutor al doctorando, asignación del director de tesis, evaluación anual del plan de investigación del doctorando, y autorización de la presentación de tesis en los programas de doctorado.

La normativa estipula la creación de Escuelas de Doctorado en las universidades para organizar los estudios y actividades del doctorado, considerando la legislación de cada Comunidad Autónoma y los Estatutos universitarios. Las Escuelas de Doctorado están registradas en el Registro de Universidades, Centros y Títulos (RUCT) ${ }^{5}$ del Ministerio de Educación, Cultura y Deporte del Gobierno de España (MECyD).

\subsection{REESTRUCTURACIÓN DE LOS PROGRAMAS DOCTORALES: ACREDITACIÓN, INTERNACIONALIZACIÓN Y TRANSFERENCIA DE CONOCIMIENTO}

La formación doctoral sufre modificaciones para converger con Europa y establecer un marco comparativo común. El cambio más significativo está en introducir una orientación formativa más centrada en dotar de competencias para la investigación que incidir exclusivamente en la formación del profesorado universitario. Así, las enseñanzas de doctorado están estructuradas en programas de doctorado, verificados por el Consejo de Universidades, acreditados evaluativamente por la ANECA (Anexo II, RD 99/2011), y coordinados por un investigador relevante que justifique tener al menos dos sexenios de investigación reconocidos y dos tesis doctorales dirigidas.

Los Programas aparecen definidos como "un conjunto de actividades conducentes a la adquisición de las competencias y habilidades necesarias para la obtención del título de Doctor" (art.2.2). La finalidad es avanzar en los aspectos formativos del doctorando que formen para la función investigadora mediante el desarrollo de líneas de investigación que concluyan en la realización de la tesis doctoral propia de unos estudios doctorales con entidad autónoma respecto de otros ciclos formativos universitarios. En esta formación doctoral es clave el vínculo con los grupos de investigación constituidos que avalen

\footnotetext{
En el RUCT pueden consultarse las Escuelas de Doctorado existentes en las Universidades Españolas y los Programas Oficiales de Doctorado registrados, https://www.educacion.gob.es/ruct/consultacentros?actual=centros
} 
líneas y proyectos de investigación que consoliden la formación del doctorado (ANECA, 2009).

Al profesorado perteneciente a los grupos de investigación se le exige para la participación en doctorado una experiencia investigadora acreditada de al menos un sexenio de investigación o méritos equivalentes a evaluar por la Comisión Académica, aspecto valorado positivamente en la investigación de Castro et al. (2012), aunque este requisito ha ocasionado la expulsión de profesorado con una trayectoria formativa en doctorado. A la clásica figura de director de tesis (art.2.6.-art.12.1., RD 99/2011) se crea la figura del tutor (art.2.7.), responsable de las actividades formativas del doctorando. Ambas figuras forman parte de la dedicación docente (art.12.3.) y tienen atribuidas una serie de funciones, entre las que destaca estimular la transición de sus doctorandos al mundo laboral y facilitar la publicación de los resultados de su investigación.

La transformación de los programas doctorales implica una armonización de los numerosos Programas departamentales e interdepartamentales existentes con anterioridad, estableciendo un número más reducido de Programas (Sevilla, 2012), que pueden desarrollarse por una universidad o conjuntamente por varias, y cuya finalidad es la formación doctoral en la investigación científica, introduciendo la posibilidad de colaboración de "aliados externos" (art.8.2., RD 99/2011), es decir, de otras entidades, organismos e instituciones con actividades de $\mathrm{I}+\mathrm{D}+\mathrm{i}$, que generen potencialidades para la investigación y la obtención de financiación, y que consoliden la formación investigadora del doctorando más allá del ámbito universitario.

Unido a ello, los programas de doctorado se reorientan para propiciar la movilidad e internacionalización de los estudios doctorales, ya sea acogiendo alumnado de otros países para la formación doctoral, auspiciando la realización de estancias de investigación doctorales de un mínimo de tres meses en universidades extranjeras, incorporando doctorandos y profesorado extranjeros en nuestras universidades o incrementado la lectura de tesis doctorales dentro de los programas que tengan el distintivo de "Mención Internacional". Además, los programas de doctorado pueden optar a una convocatoria para conseguir el sello de "Doctorado de Excelencia", hecho distintivo para aquellos programas que destacan por sus meritorios resultados. Este hecho posibilita la internacionalización del profesorado visitante y un mayor apoyo en los recursos, sobre todo en la mayor concesión de becarios de investigación (Jiménez \& Sevilla, 2016).

Por tanto, los programas de doctorado se reconfiguran para hacer hincapié en la preparación de doctores investigadores que puedan integrarse en el ámbito laboral, no vinculándose exclusivamente al mundo universitario, a la vez que pretenden reorientar las perspectivas laborales, profesionales y sociales del alumnado. Además, las directrices indican un giro en los estudios doctorales, centrado en la importancia de que los doctores deben dirigir la transferencia de conocimiento con la finalidad de contribuir a un bienestar y desarrollo de la sociedad, donde se vayan integrando en el ámbito empresarial, social y productivo, considerando sus competencias investigadoras. Es importante el vínculo entre formación doctoral, realización de la carrera investigadora y transferencia de conocimiento (Jiménez Ramírez, 2017).

La ANECA, mediante el "Programa Mención", evalúa los Programas Doctorales que optan a una Mención hacia la Excelencia, si bien esta distinción no se ha convocado desde curso académico 2011-2012. 
Este es un gran reto en la medida en que solo el 19,8\% de los doctores en activo a 31 de diciembre de 2006 estaba empleado en el sector empresas e instituciones privadas sin fines de lucro, el 44,4\% tenía empleo en el sector Enseñanza Superior y el 35,8\% en la Administración Pública (Cortina \& González, 2009). Un estudio más reciente de Benito Gil y Romera (2014, p. 14) también corrobora que "la tasa de doctores empleados en el sector privado en España, en 2009, resulta ser inferior al 16\%, lo cual da indicios de la brecha existente en términos de innovación", en la medida en que "España muestra una reducción de la tasa promedio de empleo en el sector productivo de los países miembros de la OCDE, hasta en un 50\%. El gasto privado financia en España el 43\% de la I+D, mientras que la tasa promedio de los países de la OCDE se eleva hasta el 61\%) (Benito Gil \& Romera, 2014, pp. 9-10).

\subsection{COMPETENCIAS PARA LA FORMACIÓN DOCTORAL}

Los programas de doctorado se redefinen orientando la formación doctoral hacia la investigación y la adquisición de competencias que sean transferibles a un modelo productivo dentro de una economía sostenible, que "necesita de los doctores como actores principales de la sociedad en la generación, transferencia y adecuación de la I+D+i" (RD 99/2011: 13.909). La delimitación de las competencias se realiza a partir de los "Descriptores de Dublín” (MECyD, 2005) y son las que están definidas en el Marco Español de Cualificaciones para la Educación Superior (MECES), del RD 1027/2011.

La normativa sobre el doctorado actual detalla en su art.5.1. un amplio número de competencias: comprensión sistemática de un campo de estudio y dominio de las habilidades y métodos de investigación relacionados con dicho campo; capacidad de concebir, diseñar o crear, poner en práctica y adoptar un proceso sustancial de investigación o creación; capacidad para contribuir a la ampliación de las fronteras del conocimiento a través de una investigación original; capacidad de realizar un análisis crítico y de evaluación y síntesis de ideas nuevas y complejas; capacidad de comunicación con la comunidad académica y científica y con la sociedad en general acerca de sus ámbitos de conocimiento en los modos e idiomas de uso habitual en su comunidad científica internacional; capacidad de fomentar, en contextos académicos y profesionales, el avance científico, tecnológico, social, artístico o cultural dentro de una sociedad basada en el conocimiento.

Además, la obtención del título de doctor debe también proporcionar capacitación profesional para aquellos ámbitos que requieren creatividad e innovación, adquiriendo capacidades y destrezas personales para desenvolverse en contextos en los que hay poca información específica; encontrar las preguntas claves que hay que responder para resolver un problema complejo; diseñar, crear, desarrollar y emprender proyectos novedosos e innovadores en su ámbito de conocimiento; trabajar tanto en equipo como de manera autónoma en un contexto internacional o multidisciplinar; integrar conocimientos, enfrentarse a la complejidad y formular juicios con información limitada; la crítica y defensa intelectual de soluciones (art.5.2.).

En el "XI Foro Aneca", dedicado a los estudios de doctorado, también se debatió sobre las competencias para el doctorado. Nebot (2009) identifica las competencias necesarias para el doctorado en disciplinares (demostrar comprensión en un campo de estudio), metodológicas (demostrar dominio de métodos de investigación), instrumentales (demostrar dominio de técnicas de investigación) y genéricas o transferibles. Las 
disciplinares y metodológicas se adquieren en la formación del programa de doctorado; las instrumentales, durante el desarrollo de la tesis doctoral; la complicación, en tanto, se encuentra en las competencias transferibles, que han de asumirse institucionalmente y a través de las Escuelas de Doctorado. Este reto también se percibe en la encuesta realizada a los participantes en el "XI Foro ANECA". Del análisis de sus respuesta se infiere que "las oportunidades laborales que ofrece el título de doctor son más útiles en la universidad que en la empresa" (ANECA, 2009, p. 106).

\section{LOS ESTUDIOS DE DOCTORADO EN CIFRAS}

Este apartado presenta una panorámica descriptiva de los datos derivados de la implementación de normativa sobre la configuración y el desarrollo de los estudios doctorales, utilizando las estadísticas universitarias oficiales del MECyD, el estudio de Hernández y Pérez (2015) sobre la universidad española y la información disponible en el RUCT.

La novedad principal es la creación de las Escuelas de Doctorado. En el territorio español hay 1.042 centros universitarios — facultades y escuelas-(MECyD, 2014), distribuidas en 82 universidades. De estas, 50 son universidades públicas (47 presenciales, 1 no presencial y dos especiales - UIMP y UNIA-) y 32 son universidades privadas (28 presenciales y 4 no presenciales) (Hernández \& Pérez, 2015). La totalidad de universidades no ha creado Escuelas de Doctorado. Se han establecido 55 Escuelas de Doctorado, 1 Escuela de Doctorado y Estudios de Posgrado, 1 Escuela de Posgrado y Doctorado, 2 Escuelas de Máster y Doctorado, un Centro Internacional de Posgrado y 1 Escuela de Doctorado e investigación, todas pertenecientes a universidades presenciales (públicas y privadas) (RUCT).

Si atendemos a su denominación, se ha optado por la denominación de Escuela de Doctorado. Únicamente 6 universidades han unido máster y doctorado o posgrado, incluyendo en este la formación doctoral; en 16 universidades se incluye en el nombre de la escuela el calificativo de "internacional" y en otra universidad se opta en la denominación por "Centro Internacional de Posgrado". Mayoritariamente, las Escuelas Doctorales se han configurado en universidades públicas (48), aunque también hay en universidades privadas (13). La tendencia también ha sido crear una Escuela de Doctorado por universidad, con la excepción de la Universidad de Granada que ha creado tres Escuelas Doctorales.

Según distribución por comunidades autónomas, Andalucía es la que tiene un mayor número al contar con 13 Escuelas de Doctorado, Cataluña tiene 10 Escuelas Doctorales y 1 Escuela de Posgrado y Doctorado, la Comunidad de Madrid 8 Escuelas Doctorales y 1 Escuela de Doctorado e Investigación, la Comunidad Valenciana con 6, Castilla y León con 5, Región de Murcia y Galicia con 3 cada una, Comunidad Foral de Navarra con 2, Canarias con 1 Escuela de Doctorado y 1 Escuela de Doctorado y Estudios de Posgrado, y el resto de Comunidades Autónomas con 1 Escuela Doctoral, excepto Extremadura que no consta con ningún registro ni el Principado de Asturias, aunque este último tiene un Centro Internacional de Posgrado. En función del tipo de universidad (públicaprivada), mayoritariamente las Escuelas Doctorales están en universidades públicas. Hay presencia de Escuelas Doctorales en universidades privadas en Cataluña (4 Escuelas de Doctorado y 1 Escuela de Posgrado y Doctorado), Madrid (3), Andalucía (2), y Castilla y León, Comunidad Valenciana y Región de Murcia cada una con 1 Escuela Doctoral respectivamente (Gráfico 1). 
Gráfico 1. Distribución de Escuelas Doctorales por Comunidades Autónomas

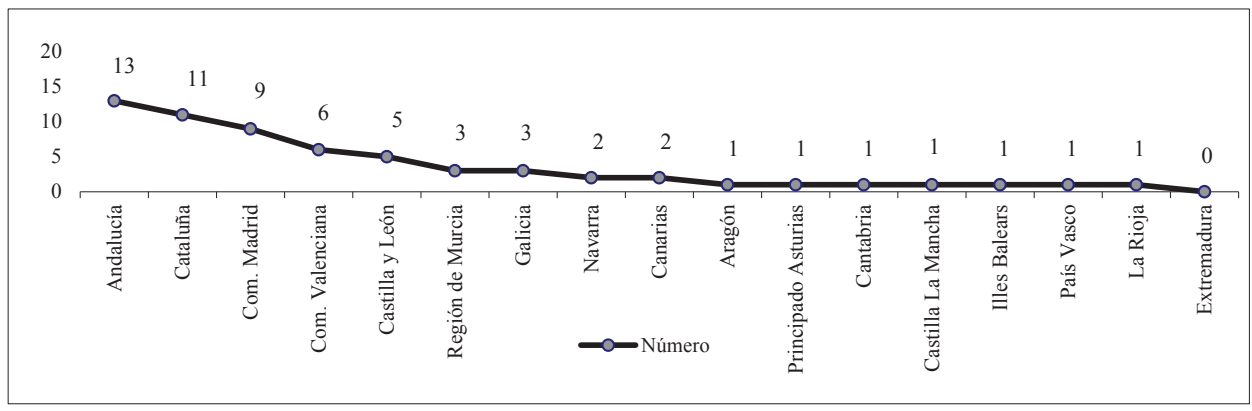

Fuente: elaboración propia a partir de los datos (RUCT)

Las Escuelas Doctorales ofertan diversos programas de doctorado evaluados y verificados por la ANECA. En el RUCT aparecen registrados 1267 Programas de Doctorado, de los que 659 son programas adaptados al EEES y están regulados por el actual RD 99/2011; los 608 programas restantes son másteres y Programas Oficiales de Doctorado y Títulos Universitarios Oficiales de Posgrado regulados por anterior normativa, que están en proceso de extinción 7 . A pesar de existir Escuelas de Doctorado registradas en el RUCT, no todas ellas tienen inscritos sus programas de doctorado, aunque si se consulta su página web, sí que ofertan programas doctorales adaptados a la actual normativa ${ }^{8}$.

También se debe indicar que del total de programas de doctorado regulados por el RD 99/2011 y registrados en el RUCT, solo algunos de ellos son compartidos por varias universidades. Los programas de doctorado están agrupados en las respectivas escuelas doctorales creadas, por ejemplo la Universidad de Granada, o están también clasificados por áreas de conocimiento (Artes y Humanidades, Ciencias de la Salud, Ciencias, Ingenierías y Arquitectura, Ciencias Sociales y Jurídicas), aunque dependiendo de la universidad pueden existir programas de doctorado exclusivos relacionados con una temática concreta como, por ejemplo, la Universidad de Cádiz, que tiene una Escuela Internacional de Doctorado en Estudios del Mar (EIDEMAR), con programas doctorales específicos sobre esta cuestión.

Sobre el número total de alumnado que cursa estudios de doctorado, las cifras indican que hay 85390 alumnos matriculados en el curso académico 2013-2014, que representan el $5,69 \%$ sobre el total de estudiantes matriculados en $1^{\circ}$ y $2^{\circ}$ ciclo, grado, máster, enseñanzas propias y doctorado, mayoritariamente en universidades públicas (Hernández \& Pérez, 2015). A pesar de ser un porcentaje minoritario quienes cursan estudios, los datos indican un incremento en el número de estudiantes matriculados en el total de universidades españolas (públicas y privadas). En concreto, se ha pasado de 74648 estudiantes de tercer ciclo en el curso académico 2008-2009 a 88732 alumnos de doctorado para el curso académico 20132014 (Hernández \& Pérez, 2015).

La revisión de los programas de doctorado está realizada a partir de la información publicada en el RUCT en octubre de 2015.

8 Por ejemplo, la Universidad Complutense de Madrid. Esta misma casuística ocurre también para algunas universidades privadas. 
La distribución de alumnado matriculado en doctorado por comunidad autónoma indica que son cuatro las que acogen a un mayor número de estudiantes: Comunidad de Madrid $(18,94 \%)$, Andalucía $(18,03 \%)$, Cataluña $(17,85 \%)$ y Comunitat Valenciana $(11,80 \%)$. El resto de comunidades descienden por debajo del 8\% (Galicia, Castilla y León, País Vasco, Región de Murcia, Aragón, Principado de Asturias, Canarias, Extremadura, Castilla La Mancha) y en otras su representación es muy minoritaria (Islas Baleares, Cantabria, Navarra y La Rioja) (Gráfico 2).

Gráfico 2. Distribución de alumnado matriculado en doctorado por comunidades autónomas

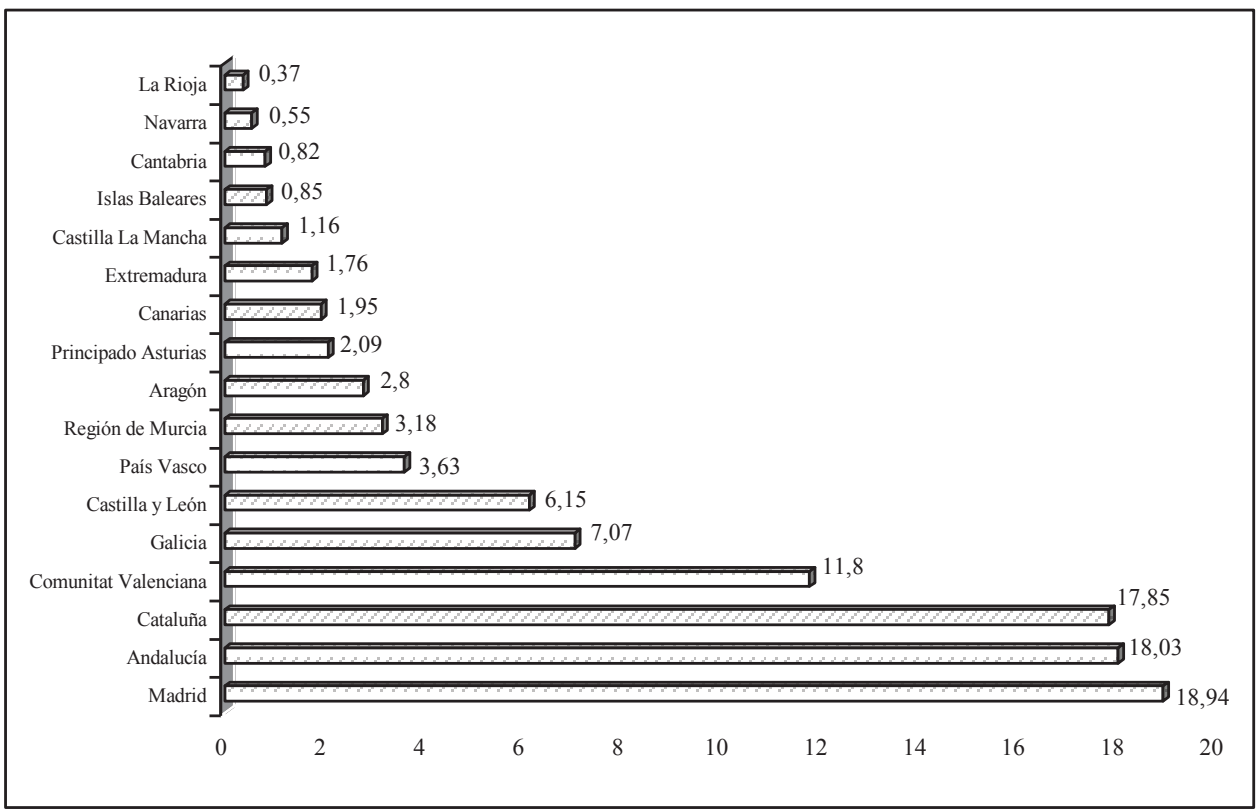

Fuente: elaboración propia a partir de los datos de Hernández y Pérez (2015).

Según el sexo, la matrícula está muy igualada entre ellos $(49,06 \%)$ y ellas $(50,94 \%)$, aunque sí hay diferenciación en la matrícula según las ramas de conocimiento y, en estas, matrícula dispar por sexo. Por ramas de conocimiento, las Ciencias Sociales y Jurídicas tienen un mayor número de matrículas (25,93\%), seguida de Ciencias de la Salud (20,95\%), Ingeniería y Arquitectura (19,46\%), Artes y Humanidades $(17,74 \%)$ y Ciencias $(15,92 \%)$. Ellas tienen mayor presencia en Ciencias de la Salud (13,06\% vs. 7,89\%), en Ciencias Sociales y Jurídicas (13,66\% vs. 12,27\%), en Artes y Humanidades (9,69\% vs. 8,05\%) y en Ciencias $(8,05 \%$ vs. $7,87 \%)$, aunque en las tres últimas con muy poca diferencia; y ellos en Ingeniería y Arquitectura (12,97\% vs. 6,49\%). Así, la diferencia más notable por sexo y rama de conocimiento se concreta en Ciencias de la Salud, que está ocupada mayoritariamente por mujeres, y en Ingeniería y Arquitectura, donde los hombres tienen una presencia muy significativa y relevante (Gráfico 3). 
Gráfico 3. Matrícula en estudios de doctorado según sexo y rama de conocimiento

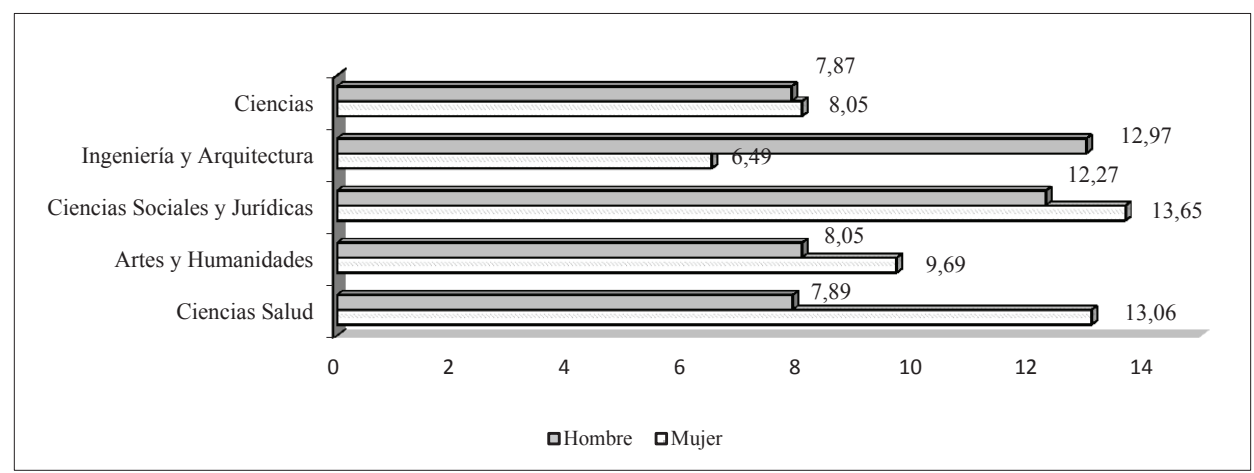

Fuente: elaboración propia a partir de los datos de Hernández y Pérez (2015).

Según la edad, el MECyD ${ }^{9}$ clasifica a los estudiante en intervalos de edad, mostrando que el acceso a la formación doctoral no tiene una edad determinada, aunque sí unos periodos más propensos para esta formación. El intervalo de edad que concentra mayor número de estudiantes es el de 25 a 30 años (41,39\%), seguido del intervalo de 31 a 40 años $(30,27 \%)$ y más de 40 años $(17,95 \%)$. Según el sexo, ellas tienen más presencia cuando son menores de 25 años (5,96\% vs. 5,06\%) y entre los 25 y 30 años (22,02\% vs. 19,37\%), existiendo mayor diferenciación para el intervalo de entre los 25 y los 30 años. Sin embargo, en los siguientes intervalos de edad ellas están presentes, pero en menor medida que ellos, para el tramo de 31 a 40 años $(14,40 \%$ vs. 15,23\%) y más de 40 años $(7,42 \%$ vs. $10,53 \%)$. Sin embargo, estos datos solo aparecen disponibles para el alumnado matriculado en doctorado conforme a la normativa del RD 99/2011.

La presentación de estas cifras evidencia un incremento progresivo en el acceso a la formación doctoral de las mujeres, aunque, precisamente, una de las ramas de conocimiento más prestigioso (Ingeniería y Arquitectura) sigue estando ocupada en mayor medida por los hombres. Villarroya et al. (2008) describen en su análisis que existe todavía un sesgo de género en la carrera científica, cuestión que se reproduce en la elección de las ramas de conocimiento entre ellos y ellas, concretamente, en Ingeniería y Arquitectura y en Ciencias de la Salud. El estudio de Cortina y González (2009), que cubre un período entre 1990 y 2006, también indica que hay más doctores hombres que mujeres salvo en edades inferiores a 35 años, en el que la participación femenina es un punto superior, aspecto que se confirma en la descripción del número de tesis defendidas por sexo que presentamos al final de este apartado.

Los estudios doctorales también tienen entre sus finalidades hacerlos atractivos para que se puedan configurar como un modelo de referencia internacional. Sin embargo, la variable nacionalidad muestra que, del total de la matrícula estatal según el RD 99/2011

9 MECyD, Estadísticas Universitarias, http://www.mecd.gob.es/educacion-mecd/areas-educacion/universidades/estadisticasinformes/estadisticas.html 
(28546) (MECyD, Estadísticas de Tesis Doctorales, 2013 ${ }^{10}$ ), la mayoría son estudiantes españoles $(77,21 \%)$, seguida de estudiantes de América Latina y Caribe (12,50\%), UE $27(5,28 \%)$ y alumnado de otras regiones de manera más minoritaria: Asia y Oceanía $(2,65 \%)$, Resto de Europa (0,92\%), Norte de África (0,70\%), Resto de África $(0,38 \%)$ y EEUU y Canadá $(0,36 \%)$. De esta información inferimos que el alumnado que cursa los estudios de doctorado es de ámbito fundamentalmente nacional, seguidos de estudiantes de América Latina y Caribe, que actualmente los estudios de doctorado no atraen de manera significativa a un número importante de estudiantes de otros países europeos o de otros contextos internacionales, y que, por ahora, los estudios de doctorado solo son un referente para Latinoamérica fundamentalmente y para algunos países europeos, pero, en menor medida (Gráfico 4). Los datos del estudio de Hernández y Pérez (2015) ofrecen información en la misma línea: el 37,48\% de alumnado de doctorado es de ámbito provincial, el 32,86\% de ámbito regional, el 13,12\% de ámbito nacional, 4,51\% proceden del entorno europeo y un $12,03 \%$ de países del resto del mundo.

Nebot (2009) reflexiona sobre movilidad internacional de los estudiantes de doctorado en Europa y establece que la dirección de los flujos de estudiantes se puede analizar siguiendo dos tendencias, desde el sur hacia el norte y desde el este hacia el oeste. La causa que justifica esta tendencia la atribuye a las "mejores condiciones de los doctorandos y doctores en los países que evidencian un mayor atractivo. Como consecuencia, el porcentaje de estudiantes extranjeros en el Reino Unido, Bélgica, Suiza y los EEUU destaca claramente sobre todos los demás sistemas universitarios" (Nebot, p. 14).

Gráfico 4. Procedencia del alumnado matriculado en doctorado en España

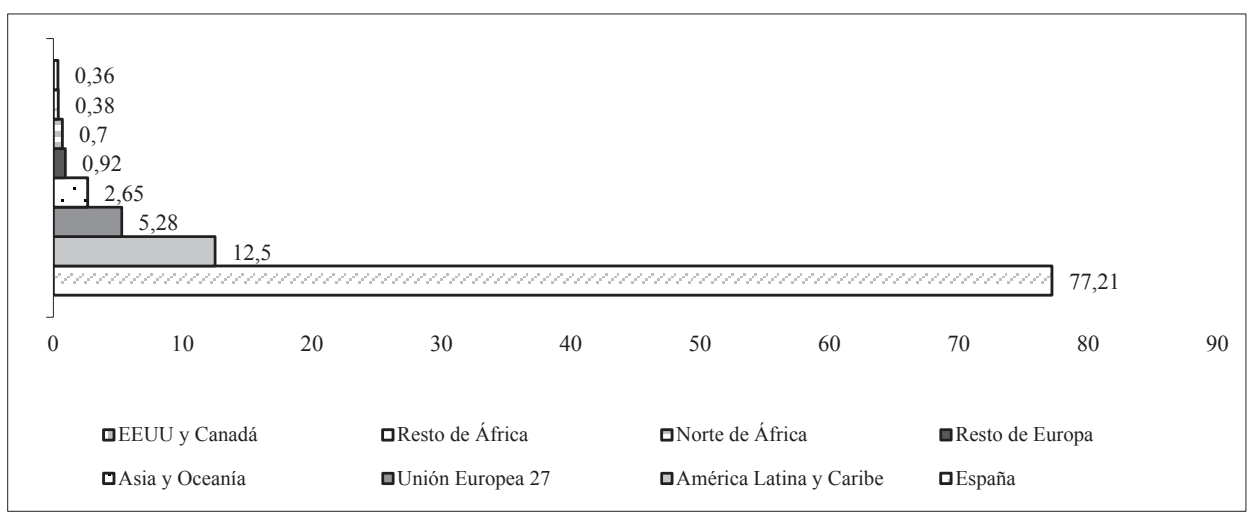

Fuente: elaboración propia a partir de los datos. MECyD, Estadísticas Tesis Doctorales (2013).

Otro aspecto clave en la formación doctoral es la vinculación del doctorando a proyectos de investigación I+D+i desde los que desarrollar su formación investigadora. El

\footnotetext{
10 MECyD, Estadísticas Tesis Doctorales, http://www.mecd.gob.es/educacion-mecd/areas-educacion/universidades/ estadisticas-informes/estadisticas/tesis-doctorales.html
} 
análisis de la convocatoria 2013 del Plan Nacional de Investigación Científica, Desarrollo e Innovación Tecnológica evidencia una concesión de proyectos de investigación limitada en la medida en que se solicitaron 3994 proyectos de investigación y se concedieron 2150 , es decir, se concedieron solo un 53,83\% sobre el total de los proyectos solicitados para las 50 universidades públicas españolas. Por comunidades autónomas, destacan Andalucía, Comunidad de Madrid y Comunidad Valenciana como aquellas que solicitaron más proyectos de investigación $(24,77 \%, 22,31 \%, 13,05 \%)$ y son esas mismas comunidades las que tienen concedidos un mayor número de proyectos científicos $(18,10 \%, 19,20 \%$, $11,77 \%$ respectivamente), junto con Cataluña, que solicitó un menor número de proyectos de los que finalmente tiene concedidos (6,03\% vs. 14,24) (Hernández \& Pérez, 2015). Estas 4 comunidades tienen más del $63 \%$ de proyectos de investigación concedidos; el resto de comunidades tiene una menor presencia tanto en solicitud de proyectos como en la concesión de los mismos.

La distribución del total de proyectos de investigación $\mathrm{I}+\mathrm{D}+\mathrm{i}$ por ramas de enseñanza para la convocatoria del 2013 muestra un mayor porcentaje de solicitudes para las áreas de Humanidades y Ciencias Sociales $(32,28 \%)$ y para las Tecnologías de la Producción y las Comunicaciones (28,42\%), seguido de Ciencias de la Vida y Agroalimentación $(21,75 \%)$ y de Medio Ambiente y Recursos Naturales (17,55\%). Sin embargo, los proyectos concedidos son mayores para Tecnologías de la Producción y las Comunicaciones $(31,21 \%)$, seguidos de Humanidades y Ciencias Sociales $(20,21 \%)$ y, muy equiparadas, las áreas de Ciencias de la Vida y Agroalimentación y Medio Ambiente y Recursos Naturales (Tabla 1).

Tabla 1. Proyectos de investigación por áreas temáticas, solicitados y concedidos, 2013

\begin{tabular}{|l|c|c|c|c|}
\hline \multicolumn{1}{|c|}{ ÁREAS TEMÁTICAS } & \multicolumn{2}{c|}{ SOLICITADOS } & \multicolumn{2}{c|}{ CONCEDIDOS } \\
\hline Humanidades y Ciencias Sociales & 1.289 & 32,28 & 628 & 20,21 \\
\hline Medio Ambiente y Recursos Naturales & 701 & 17,55 & 423 & 19,67 \\
\hline Ciencias de la Vida y Agroalimentación & 869 & 21,75 & 428 & 19,91 \\
\hline Tecnologías de la Producción y las Comunicaciones & 1.135 & 28,42 & 671 & 31,21 \\
\hline \multicolumn{1}{|c|}{ TOTAL } & 3.994 & $100 \%$ & 2.150 & $100 \%$ \\
\hline
\end{tabular}

Fuente: elaboración propia a partir de los datos de Hernández y Pérez (2015).

Sobre el gasto en I+D, Hernández y Pérez (2015) indican que España, a partir del 2008 y con la llegada de la crisis económica, se ha desligado de la tendencia de los países más competitivos que han dirigido sus prioridades hacia "el crecimiento sostenido del esfuerzo del gasto público y privado en I+D [...] incluso en momentos en los que se producía recesión en sus economías [...] precisamente como estrategia para la salida de la crisis" (p. 85). Siguen analizando como en "España el gasto en I+D ha retrocedido a niveles inferiores a 2007" (Hernández \& Pérez, 2015, p. 86), donde la "reducción se ha producido esencialmente en el sector Empresas, manteniéndose en el sector de la Administración pública" (Hernández \& Pérez, 2015, p. 86), precisamente porque las universidades han 
sido capaces de incrementar ligeramente el gasto en I+D manteniendo los proyectos y programas de investigación gracias a los recursos propios generados por remanentes de la actividad de I+D. Benito et al. (2014) destacan la relevancia de la inversión en I+D+i como elemento clave para el empleo de los doctores y la transferencia de conocimiento.

Otro aspecto interesante sobre el doctorado es analizar el número de tesis doctorales concluidas relacionándolas con los tramos de edad, sexo, ámbitos de conocimiento y nacionalidad. Al respecto, es posible indicar que el número de tesis doctorales aprobadas también va en incremento, desde las 7830 tesis en el año 2008 a las 10889 defendidas en el $2013^{11}$, mayoritariamente en universidades públicas (94,45\%) (Gráfico 5). El estudio de Benito et al. (2014) corrobora este incremento progresivo desde el curso académico 20012002.

Gráfico 5. Evolución del número de tesis defendidas y aprobadas por curso académico en España

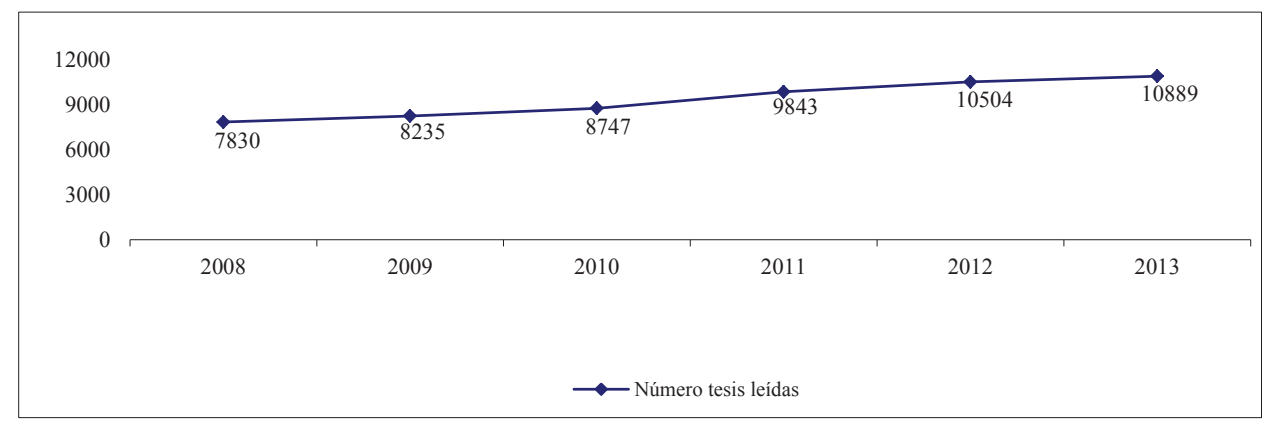

Fuente: elaboración propia a partir de los datos del TESEO (MECyD).

Por tramos de edad, entre los 30 y 34 años es el periodo en que más tesis se defienden $(35,65 \%)$, seguido del tramo entre 24 y 29 años $(18,91 \%)$, de 35 a 39 años $(17,79 \%)$ y el resto de grupos de edad con una participación menor. Por sexo, las lecturas de tesis están muy equiparadas, ligeramente superior para ellos (50,77\% vs. 49,23\%). La única diferencia está en los tramos de edad. Ellas defienden mayoritariamente entre los 30-34 años (18,98\%), seguida del tramo 24-29 años (10,54\%). Ellos se demoran un poco más y el mayor número de lecturas se realiza en el período entre los 30-34 años (16,67\%), seguido de 35-39 años (9,32\%) y de 8,37\% para el tramo de 24 a 29 años. Además, el número de tesis defendidas después de los 40 años es bastante más elevada para ellos y desciende para ellas.

Las tesis doctorales defendidas por ámbitos de estudios muestran que en las universidades públicas el mayor número de tesis aprobadas fue en el ámbito de Ciencias e Informática (35,91\%), seguido por el ámbito de las Ciencias Sociales y Derecho (16,56\%), Salud y Servicios Sociales (13,85\%), Artes y Humanidades (11,35\%), Ingeniería, Industria y Construcción (10\%), Educación (4,2\%), Agricultura y Veterinaria (2,07\%), Servicios

11 Fuente: S.G. de Coordinación y Seguimiento Universitario. TESEO. MECyD, http://www.mecd.gob.es/educacion-mecd/ areas-educacion/universidades/estadisticas-informes/estadisticas/tesis-doctorales.html 
$(0,51 \%)$. Por sexo, los datos están muy equiparados, aunque las diferencias se vuelven a mostrar favorables para ellos en Ciencias e Informática $(18,65 \%$ vs. 17,27\%) y en Ingeniería, Industria y Construcción (6,51\% vs. 3,49\%), y para ellas en Salud y Servicios Sociales $(8,50 \%$ vs. $5,35 \%)$ y en Educación (2,57 vs. 1,63\%) (Gráfico 6).

Gráfico 6. Tesis defendidas y aprobadas en España por ámbitos de estudio según sexo en universidades públicas

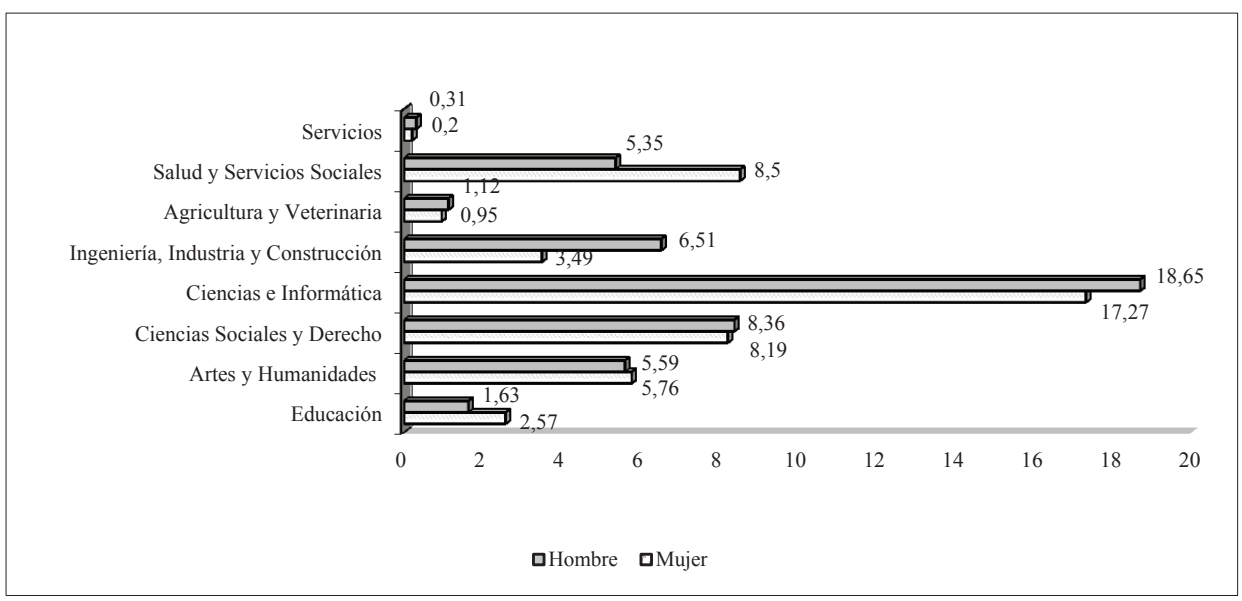

Fuente: elaboración propia a partir de datos del TESEO.

Como hemos descrito, el acceso a los estudios doctorales por ramas de conocimiento manifiesta todavía una brecha de género, presente en la obtención del máximo grado académico. Si bien inicialmente existe un equilibrio en la obtención del grado de doctor en España, la diferenciación sigue existiendo y es aún muy evidente en las ramas de conocimiento que están relacionadas con una perspectiva de socialización diferenciada de género y que, además, poseen un mayor reconocimiento social y prestigio en la consolidación de una carrera investigadora de éxito. Una de las investigaciones explicita que

sí se llega a un cierto nivel de igualdad en las primeras huellas de las carreras académicas y científicas, pero esta igualdad no rompe en otros tipos de segregaciones como son las que se dan entre los ámbitos de conocimiento ni entre las relaciones segregadas que se establecen entre los hombres y las mujeres en el mundo académico. (Borrás, 2008, p. 132).

La diferenciación de género también está presente en el porcentaje de mujeres en funciones de dirección, que todavía es bajo (Grifoll, 2009).

En consonancia con la procedencia del alumnado matriculado en doctorado, el alumnado español es el que mayoritariamente defiende las tesis en nuestras universidades (75,13\%), seguido de América Latina y el Caribe (14,60\%), UE 27 (6,50\%), Asia y Oceanía $(1,27 \%)$, Norte de África $(1,07 \%)$, Resto de Europa $(0,73 \%)$, Estados Unidos 
y Canadá (0,38\%), y Resto de África (0,32\%). Si se analizan datos de años anteriores, podemos observar que se incrementa el número de estudiantes extranjeros que defienden tesis en España y que la tendencia es de alumnado de Latinoamérica y el Caribe, seguido de estudiantes de la UE 27.

\section{CONCLUSIONES FINALES Y DISCUSIÓN}

Las reformas iniciadas en la universidad para adaptarse a los planteamientos del EEES han conllevado transformaciones de relevancia en los estudios de tercer ciclo. Los estudios de doctorado no han estado ajenos a los cambios de normativa para converger con Europa en el establecimiento de una red de formación en investigación para los estudios doctorales. Esta etapa es clave para la formación en la medida en que se crea un vínculo entre docencia, investigación, universidad y sociedad (Castro et al., 2010), con la finalidad de generar transferencia del conocimiento a la sociedad por parte de los doctores.

Estos cambios han sido burocráticos y complejos en la medida en que se han sucedido distintos RD para adaptar el doctorado a los planteamientos europeos. El RD 99/2011 es el que establece las directrices básicas para los estudios de tercer ciclo, creando, como principal innovación, las Escuelas de Doctorado, delimitadas como una entidad para gestionar y coordinar todo lo relativo al doctorado así como al funcionamiento de los programas de doctorado. Se han instituido Escuelas de Doctorado en casi todas las comunidades autónomas, aunque destacan Andalucía, Cataluña, Madrid y Comunidad Valenciana. Los programas de doctorado configurados desde las escuelas doctorales tienen una orientación más centrada en la investigación y en la formación en competencias que sean transferibles al entorno social, no siendo ya la finalidad exclusivamente la de formar profesorado universitario.

Este cambio de perspectiva para los estudios de doctorado supone un reto porque estos deben transformarse para unas nuevas exigencias que demandan establecer unos lazos más estrechos entre investigación y organismos de $\mathrm{I}+\mathrm{D}+\mathrm{i}$ que transfieran el conocimiento a la sociedad, con la finalidad de formar doctores altamente cualificados en investigación y producción científica y tecnológica, representando un valor estratégico de primera magnitud para su inserción en el sector productivo (Benito et al., 2014). Junto a ello, no todas las áreas de conocimiento, por ejemplo las Ciencias Sociales, tienen la misma experiencia para aproximarse a ese cambio de orientación, al carecer de una trayectoria con empresas e instituciones sociales en las que desarrollar el trasvase de conocimiento (Jiménez y Sevilla, 2016). Por ello, las Escuelas de Doctorado, las universidades y los organismos y empresas deben aunar esfuerzos para encauzar este reto.

Los nuevos planteamientos para los estudios doctorales implican también una reestructuración de los programas de doctorado. El número de programas departamentales e interdepartamentales existentes se reduce y se les dota de unas características distintas: programas verificados y acreditados por la ANECA; formación en competencias disciplinares, metodológicas, instrumentales, investigadoras y transferibles en un ámbito científico del conocimiento (Nebot, 2009); necesidad de promover la movilidad e internacionalización del doctorado, tanto de profesorado como de los doctorandos; y acreditación de sexenios de investigación para participar como tutor, profesor y/o director en los estudios de doctorado. 
Esta reconfiguración de los programas de doctorado presenta también algunos desafíos. Por un lado, conlleva que el doctorando se vincule a grupos y líneas de investigación para desarrollar su actividad investigadora y concluir la tesis doctoral, a pesar de que los grupos de investigación "tienen un estatus poco o nada definido" (Nebot, 2009, p. 15). Por otro lado, la consolidación de los grupos de investigación podría mejorarse incrementando la inversión del gasto público y privado en I+D (Hernández \& Pérez, 2015) y la financiación pública de la universidad (Gutiérrez-Solana, 2010), redundando este hecho en una concesión de proyectos de I+D+i más generosa y no tan limitada como la actual.

Este proceso permitiría generar conocimiento científico contando con los recursos económicos y humanos obtenidos que, a su vez, permita que los doctorandos contribuyan a la producción y difusión del conocimiento generado desde la universidad y sitúe también al profesorado en disposición de publicar los resultados de su investigación para optar a la convocatoria de sexenios, requisito imprescindible para participar en el doctorado. Finalmente, la transferencia de conocimiento y la transición al mundo laboral más allá de la universidad, implica la prioridad de establecer vínculos entre universidades, empresas e instituciones privadas de I+D+i (Cortina \& González, 2009; Benito et al., 2014), que sitúen a los doctorandos en otras esferas del mercado laboral y que generen innovación.

Abordar estas limitaciones es clave si se pretende que los estudios doctorales incrementen el número de alumnado matriculado, sean competitivos en su vinculación con el mundo laboral y supongan un atractivo internacional para los estudiantes. El análisis presentado muestra que, a pesar del aumento en el número de alumnado realizando estudios doctorales, la formación en estudios de tercer ciclo la realizan una minoría (Hernández \& Pérez, 2015) en universidades públicas básicamente. Ese alumnado procede mayoritariamente del ámbito estatal, seguido del colectivo de Latinoamérica y del Caribe y, en menor medida, de otras nacionalidades. Estos datos indican que, por ahora, la internacionalización del doctorado no se ha conseguido, existiendo flujos de estudiantes fundamentalmente "desde el sur hacia el norte y desde el este hacia el oeste" (Nebot, 2009, p. 14), es decir, hacia países donde existen mejores condiciones para los doctorandos y los doctores.

Los datos sobre el doctorado muestran una distribución desigual por comunidad autónoma, siendo la Comunidad de Madrid, Andalucía, Cataluña y Comunidad Valenciana las que tienen la gran mayoría del alumnado de doctorado. En función del sexo, existe una equiparación entre ellos y ellas. Sin embargo, la elección del doctorado por ramas de conocimiento muestra diferencias en Ciencias de la Salud, ocupada mayoritariamente por ellas, y en Ingeniería y Arquitectura, con un predominio masculino. El estudio de Villarroya et al. (2008) también evidencia que la carrera científica tiene un sesgo de género que reproduce desigualdades en la elección de las ramas de conocimiento del doctorado. Al igual que el "Informe del Consejo Superior de Investigaciones Científicas" (2015), que ofrece información, entre otras, sobre la distribución desigual en función del sexo del personal científico por áreas de investigación, evidenciando que esto también es un reto social.

\section{REFERENCIAS BIBLIOGRÁFICAS}

Agencia Nacional de Evaluación de la Calidad y Acreditación. (2008). Los nuevos títulos de Máster y la competitividad en las Universidades. Madrid: Autor. 
Agencia Nacional de Evaluación de la Calidad y Acreditación. (2009). El Doctorado: logros y desafíos. Madrid: Autor.

Benito, M., Gil, P., \& Romera, R. (2014). El empleo de los doctores en España y su relación con la $I+D+i$ y los estudios de doctorado. Gran Canaria: Conferencia de Estudios Sociales de Universidades Españolas.

Borrás, V. (2008). Introducción. En M. J. Izquierdo (Dir.), Actas del I Congreso Internacional sobre sesgo de género y desigualdades en la evaluación de la calidad académica (pp. 131-134). Barcelona: Universidad Autónoma de Barcelona-Observatorio de Igualdad.

Castro, A, Guillén, A., Quevedo, R., Bermúdez, Ma P., \& Buela-Casal, G. (2012). Doctoral Schools in Spain: Suggestions of Professors for their Implementation. Revista de Psicodidáctica, 17(1), 199-217.

Castro, A., Guillén, A., Quevedo, R., Ramiro, M. T., Bermúdez, M. P., \& Buela-Casal, G. (2010). Las Escuelas Doctorales: evolución histórica, características y aspectos relevantes para su consolidación en España. Aula Abierta, 38(2), 17-28.

Consejo Superior de Investigaciones Científicas. (2015). Informe Mujeres Investigadoras 2015. Madrid: CSIC.

Cortina, F., \& González, B. (2009). La encuesta sobre recursos humanos en Ciencia y Tecnología. Un instrumento de análisis de la situación de los doctores en España y su movilidad. En Agencia Nacional de Evaluación de la Calidad y Acreditación (Ed.), El doctorado: logros y desafíos (pp. 21-30). Madrid: ANECA.

Grifoll, J. (2009). La educación de posgrado (Doctorado) desde la perspectiva del estudiante. En Agencia Nacional de Evaluación de la Calidad y Acreditación (Ed.), El doctorado: logros y desafios (pp. 79-88). Madrid: ANECA.

Gutiérrez-Solana, F. (2010). Políticas universitarias para una nueva década: problemas y oportunidades. La Cuestión Universitaria, 6, 4-11.

Haug, G. (2008). Legislación europea y legislaciones nacionales. Revista de Educación, $\mathrm{n}^{\mathrm{o}}$ extraordinario, 285-305.

Hernández, J., \& Pérez, J.A. (2015). La Universidad Española en cifras. Madrid: CRUE.

Huber, G.L. (2008). Aprendizaje activo y metodologías educativas. Revista de Educación, n $^{\circ}$ extraordinario, 59-81.

Jiménez Ramírez, M. (2017). Los nuevos estudios de Doctorado en España: avances y retos para su convergencia con Europa. Revista Iberoamericana de Educación Superior. En prensa.

Jiménez, M., \& Sevilla, D. (2016). Doctoral Studies in Spain. In C. Cappa, \& D. Palomba (Comps.), Doctoral Studies in Europe. Rome: Aracne Editrice.

Ley Orgánica 6/2001, de 21 de diciembre, de Universidades, modificada por la Ley Orgánica de Universidades 4/2007, de 12 de abril. Boletín Oficial del Estado, (89), 16.241-16.260.

Ministerio de Educación, Cultura y Deporte. (2014). Datos y cifras del sistema universitario español. Curso 2014-2015. Madrid: Gobierno de España. Recuperado el 20 de diciembre de 2015 desde http://www.mecd.gob.es/dms/mecd/educacion-mecd/areas-educacion/universidades/ estadisticas-informes/datos-cifras/Datos-y-Cifras-del-SUE-Curso-2014-2015.pdf

Ministerio de Educación, Cultura y Deporte. (2005). Informe sobre un marco de cualificaciones para el Espacio Europeo de Educación Superior. Comisión Europea. Recuperado el 15 de diciembre de 2015 desde http://www.mecd.gob.es/dctm/ministerio/educacion/mecu/normativa/marco-es. pdf?documentId=0901e72b806a6c63

Nebot, I. J. (2009). Introducción. El desafío de los programas de doctorado. En Agencia Nacional de Evaluación de la Calidad y Acreditación (Ed.), El doctorado: logros y desafíos (pp. 11-20). Madrid: ANECA.

Real Decreto 56/2005, de 21 de enero, por el que se regulan los estudios universitarios oficiales Posgrado. Boletín Oficial del Estado, (21), 2.846-2.851.

Real Decreto 1393/2007, de 29 de octubre, por el que se establece la ordenación de enseñanzas 
universitarias oficiales. Boletín Oficial del Estado, (260), 44.037-44.048.

Real Decreto 1027/2011, de 15 de julio, por el que se establece el Marco Español de Cualificaciones para Educación Superior. Boletín Oficial del Estado, (185), 87.912-87.918.

Real Decreto 99/2011, de 28 de enero, por el que se regulan las enseñanzas oficiales de Doctorado. Boletín Oficial del Estado, (35), 13.909-13.926.

Real Decreto 534/2013, de 12 de julio, por el que se modifican los Reales Decretos 1393/2007; 99/2011y 1892/2008. Boletín Oficial del Estado, (167), 52.159-52.161.

Sevilla, D. (2012). Doctoral Studies in Spain. The Arduous Shift from Legal and Bureaucratic Control to Research Quality and Social Relevance. In D. Palomba, \& C. Cappa (Eds.), Doctoral Studies in Europe (pp. 105-127). Rome: Aracne Editrice.

Vázquez, J. A. (2008). La organización de las enseñanzas de grado y postgrado. Revista de Educación, $\mathrm{n}^{\circ}$ extraordinario, 23-39.

Villa, A., \& Ruiz, M. (2004). La Red de Educación y el Espacio Europeo de Educación Superior. Revista Interuniversitaria Formación Profesorado, 18(1), 21-27.

Villarroya, A., Barrios, M., Borrego, À., \& Frías, A. (2008). La obtención del Doctorado en España: un análisis de género. En M. J. Izquierdo (Dir.), Actas del I Congreso Internacional sobre sesgo de género y desigualdades en la evaluación de la calidad académica (pp. 159-168). Barcelona: Universidad Autónoma de Barcelona-Observatorio de Igualdad. 